\title{
To treat or not to treat, that really is not the question
}

\author{
Guoqiao Wang, PhD
}

Gary R. Cutter, PhD

$\mathrm{F}$

ew patients come to physicians with the expectation that they won't be treated. They have a problem and expect that a good physician will provide some sort of relief, which to them often means a drug or treatment. While physicians may often question the benefits of a particular therapy, how to decide whether one therapy is as good as another is a challenge. From a researcher's perspective, the question is: How do you know one treatment is as good as another? Consider the situation in which payers say they will only

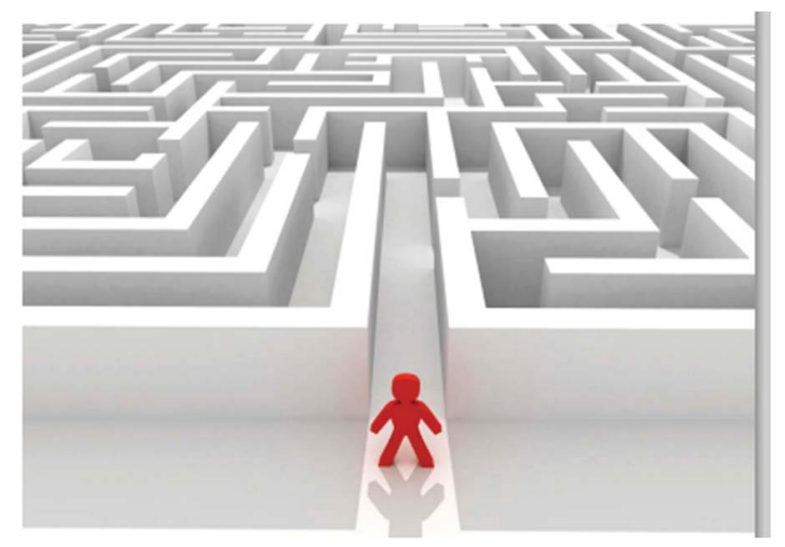
pay for a generic drug. How do you-and, in fact, they-know that the generic drug is really as good as the brand name drug? Mostly likely the generic drug costs considerably less than the brand name drug, so one might be suspicious that the payer is merely trying to maximize profit. The payer is trying to reduce costs but relies on the fact that the US Food and Drug Administration (FDA) has provided approval for this generic drug; therefore, it must be as good as the brand name drug.

Intellectually, this makes sense. If one drug is proven to have the same benefits and risks as another, then why would it matter which drug is given? Is a generic drug then identical to a brand name drug? The answer, of course, is no. But how is sameness defined? Clearly snakes and humans are different (at least in physical terms). They are not the same since at a minimum snakes don't have legs. But horses have legs and they are not humans. Two humans are not the same and usually we can quantify or qualify how they are different (unless they are identical twins). So there is a spectrum of "sameness" depending on the question. Sameness of treatments can be thought of with several levels of comparison: (1) one treatment may be superior to another, (2) one treatment may be inferior to another, (3) one treatment may be equivalent to another, and (4) one treatment may not be worse than another but just may not be better.

Each of these 4 scenarios is important in medicine. Superiority and inferiority are features distinguishing one treatment from another. Drugs are often licensed based on being superior to either a placebo or an active drug with comparable effects. However, when 2 treatments both work, how is the decision made that they are the same?

University of Alabama, Birmingham, AL.

Funding information and disclosures are provided at the end of the article. Full disclosure form information provided by the authors is available with the full text of this article at Neurology.org/cp.

Correspondence to: cutterg@uab.edu 
Table 1 Two batches of vials with the same mean volume

\begin{tabular}{|c|c|c|c|c|c|c|c|c|c|c|c|}
\hline & Vial 1 & Vial 2 & Vial 3 & Vial 4 & Vial 5 & Vial 6 & Vial 7 & Vial 8 & Vial 9 & Vial 10 & Average \\
\hline Batch 1 & 20 & 18 & 16 & 14 & 12 & 10 & 8 & 6 & 4 & 2 & 11 \\
\hline Batch 2 & 11.1 & 11.1 & 11.1 & 11 & 11 & 11 & 11 & 10.9 & 10.9 & 10.9 & 11 \\
\hline
\end{tabular}

When a drug or treatment dosage is formulated, equivalence is expected. For example, if insulin is given to a patient with diabetes in a prefilled syringe, the amount of insulin in each syringe must be neither too little nor too much. In other words, each syringe is expected to be equivalent to any other syringe, i.e., the principle of equivalence. When patient education is provided as an aid to a patient compliance, patients are expected to do as well or better than they would have if educational training had not been given. Rarely is it thought that such education could be harmful to a treatment regimen, i.e., the principle of noninferiority.

Noninferiority in drug trials implies that an alternative drug or treatment is as good as or better than a standard drug or treatment. Conceptually, this is easy to understand, but the obvious question arises when there is more than one treatment option. The complexity of the research that tries to address this simple and practical situation is much more difficult than the common null hypothesis of a superiority trial. ${ }^{1}$

To begin to build an understanding of noninferiority trials, let's start with the concept of equivalence. In the insulin syringe example, there are major consequences to using vials that are not equivalent. If the dose of insulin given is doubled or halved, there can be substantial effects on the patient. Thus, when 2 doses are said to be "equivalent," there is some notion of sameness in mind. But how similar is similar? If the desired dosage was $10 \mathrm{~mL}$ of insulin and 1 vial had $10.00 \mathrm{~mL}$ and another had $10.01 \mathrm{~mL}$, we would probably be content that these vials are equivalent. Thus, we have in our minds some tolerable difference we would accept and still call the dosage in the vials equivalent. This tolerable difference is called the margin of tolerability or tolerable margin. If we know what that margin is, we can assess whether 2 vials are the same or different. While 2 individuals may agree on what is clearly different, finding a value that they would agree is the same is a bit more difficult.

If I produce the vials from my basement and show you that 2 are within $0.05 \mathrm{~mL}$ of each other, would you be satisfied that all the vials in my basement are equivalent? You have some data on their equality, but you might want to examine a few more details to increase your confidence. Indeed, if you work for the FDA, you would want the manufacturer of the vials to prove to you that they contain equivalent amounts of insulin. How can this be done in a rigorous research manner?

Suppose I present you with the results of a study that shows that the mean of 10 vials is not significantly different from 10 . Does that prove the sameness of the vials? It does NOT. Consider the vial amounts in table 1.

You can see that the average amount of insulin in the vials is $11 \mathrm{~mL}$ in both batches, but clearly the 2 batches are not the same. Batch 1 has much more variability, with 3 of 10 vials having more than $15 \mathrm{~mL}$. This would make you wonder whether they are equivalent. Thus, to say that 2 things are equivalent we want to say more than that their means are the same.

We also know that for any difference in mean volume we can find a sample size that will say 2 groups are statistically significantly different. For example, suppose the mean volume in batch 1 was $11.00 \mathrm{~mL}$ with an SD of $1 \mathrm{~mL}$, whereas batch 2 had a mean volume of $11.05 \mathrm{~mL}$ with an SD of $1 \mathrm{~mL}$. If we examined 3,100 vials from batch 1 and 3,100 vials from batch 2, our $t$ test for the difference between the means would be statistically significantly different $(t=[11.05-11.00] /$ sqrt $[1 / 3,100+1 / 3,100]=1.97$, which results in a 2 -tailed $p$ value of $<0.05$ ).

Thus, we would say that the volume of insulin in batch 1 was statistically significantly different from that in batch 2. While no one would sample 3,100 vials from each batch, going 
Table 2 Two batches of vials, different but not statistically significantly different

\begin{tabular}{|lllllllllllll} 
& Vial 1 & Vial 2 & Vial 3 & Vial 4 & Vial 5 & Vial 6 & Vial 7 & Vial 8 & Vial 9 & Vial 10 & Average & SD \\
Batch 1 & 20 & 20 & 18 & 14 & 12 & 10 & 6 & 6 & 2 & 2 & 11 & 6.94 \\
Batch 2 & 18 & 14 & 11 & 8 & 6 & 5 & 4 & 3 & 2 & 1 & 7.2 & 5.55
\end{tabular}

through this process would tell us that the vials are not the same. Yet clinically we might be comfortable that they are essentially the same.

So, in the first example the means were equal, but we were not convinced that the vials were the same because of their variability, whereas in the second example the means were statistically significantly different, but we thought that the difference was probably insignificant clinically.

Consider another scenario, represented in table 2. Here the mean volume in batch 1 is $11 \mathrm{~mL}$ compared with $7.2 \mathrm{~mL}$ in batch 2, with SDs of 6.94 and 5.55, respectively. The difference is $3.8 \mathrm{~mL}$, more than a $30 \%$ difference on average, but this is NOT a statistically significant difference $(p=0.07)$. As demonstrated by this scenario, the absence of a statistically significant difference is also in and of itself not evidence for equivalence.

So, how can equivalence be convincingly demonstrated clinically?

What features of the insulin vial examples bothered us? In the first example (table 1), the variability was the issue; in the second example, there was a difference but of a magnitude that was tolerable; and in the third example (table 2), there was not a statistically significant difference but it seemed clear that the batches were different.

One key to establishing equivalence is to determine how different 2 things need to be before we accept that they are different. To put it another way, what difference will we tolerate? Given a tolerable difference, we can design a study to show that 2 things are equivalent.

To do this, we have to show that for the tolerable difference, the vial does not contain significantly more than it should or significantly less than it should. How can we do this? We need Two One-Sided Tests ("TOST"). In the first test, we hypothesize that the mean volume in batch 1 vials is higher than that in batch 2 vials; that is, we assume the null hypothesis that we were not able to produce an equivalent average vial. We hope to reject this null hypothesis that the batch 1 vials have greater volume, concluding in the first of our 2 tests that it is not true that batch 1 vials have significantly more volume than batch 2 vials.

We then perform a second test in our TOST approach and assume that the batch 1 vials have less volume than the batch 2 vials. Again, we seek to reject this assumption that the batch 1 vials have less volume. If both tests show that both null hypotheses are rejected (that is, the vials do not have a higher volume AND they do not have a lower volume), then the volume of the vials must be equivalent.

Graphically, the figure shows a set of trial outcome results for superiority, inferiority, equivalence, and noninferiority trials. If a difference between 2 treatments lies in the blue area on the far right, we would declare one treatment superior to the other. Similarly, if the difference is in the blue area to the far left, we would say that one treatment is inferior to another. If the difference (and its 2-sided 95\% confidence interval [CI]) falls in the red area in the center, we would say that the 2 treatments are equivalent, which is the same as performing our TOST. If the 1-sided $95 \% \mathrm{CI}$ is contained in the area to the left of the rightmost blue area, then we have demonstrated noninferiority.

In an attempt to clarify this issue, let's consider an example. Suppose that we want to show that a generic drug for multiple sclerosis (MS) ("BARC") is noninferior to one of the mainline drugs ("CRAB"). We believe that the percentage of patients with confirmed Expanded Disability Status Scale progression is the appropriate outcome variable upon 


\section{Equivalence trials are useful to provide evidence so that one can trust the makeup and dosing of the drugs from one manufacturer to another or from one batch to another.}

which to compare the new drug (BARC) with the mainline drug (CRAB). We could try to show that $B A R C$ is superior to $C R A B$, but if it is a generic version of CRAB, we would have no reason to think it is superior. It could be, but it is unlikely that someone would risk the millions of dollars required for a study to prove that. However, they might go for a noninferiority trial.

So, as noted earlier (in discussing noninferiority trials), we start with the null hypothesis that BARC is inferior to or worse than CRAB. Now we have to consider or understand what is meant by tolerable difference. What is a worse progression rate? If the usual progression rate in a 2 -year study is, for example, $15 \%$, then what is worse? In this instance, there are several ways to determine a tolerable difference, but all are ARBITRARY. One is the Delphi technique, in which various clinicians are polled and give their views on what is a difference. A more quantitative way is to consider the difference between an active drug and the placebo used in the studies before the drug was licensed. Suppose that in the study that led to the approval of $\mathrm{CRAB}, \mathrm{CRAB}$ achieved a $25 \%$ reduction in confirmed progression over that achieved by placebo. This implies that the placebo rate was $0.15 / 0.75=0.20$, or $20 \%$. That means that the absolute difference between CRAB and placebo in that study was 5\%. A tolerable difference would seem to be at least half of this absolute difference, and it is commonly set at one-third of the difference, so $0.05 / 3=0.0167$ would be the tolerable difference.

Given this tolerable difference, we design a study to show that the effect of BARC is not worse than that of CRAB + the previously established tolerable difference $(15 \%+1.67 \%$, or $16.67 \%$ or higher). Our null hypothesis is that BARC has a progression rate of $16.67 \%$ or higher, so we design a study to show that BARC has a statistically significantly better effect

Figure Visualizing superiority, inferiority, equivalence, and noninferiority trials

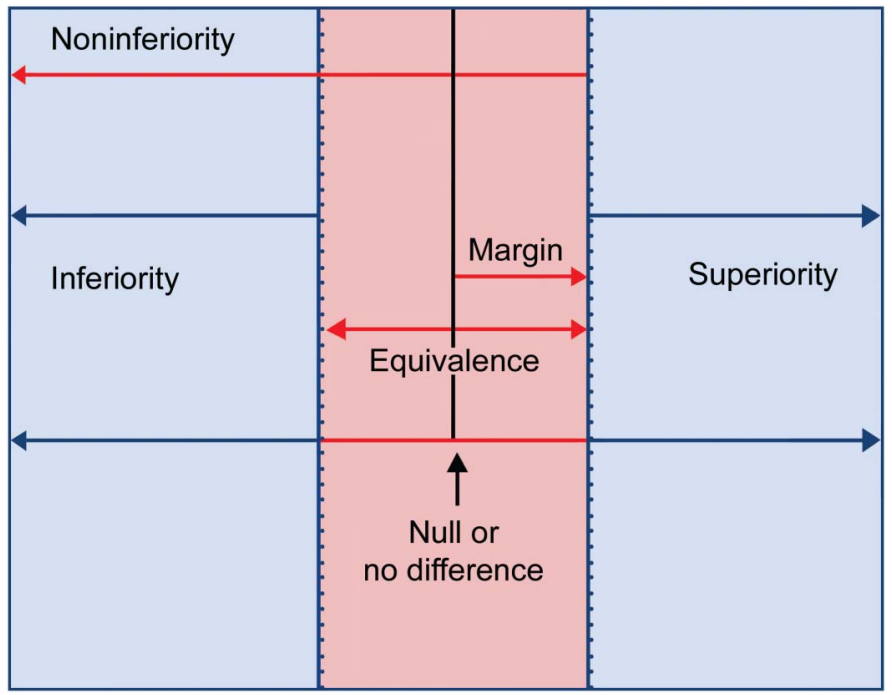




\section{Noninferiority trials can provide comparisons} between drugs that are thought to be equal in effectiveness, but they require added planning and decision making.

than that. If we believe that CRAB and BARC are truly equivalent, then we can compute the sample size necessary to establish noninferiority between them.

When the sample size in each group is 5,674, a 2-group large-sample normal approximation test of proportions with a 1 -sided 0.05 significance level will have $80 \%$ power to reject the null hypothesis that BARC is inferior to CRAB (e.g., the difference in proportions, $\mathrm{p}_{\mathrm{T}}-\mathrm{p}_{\mathrm{S}}$, is 0.017 or greater) in favor of the alternative hypothesis that the proportions in the 2 groups are noninferior, assuming that the expected difference in proportions is 0 and the proportion in the standard group is 0.15 .

Of course, this required an enormous sample size $(5,674 \times 2=11,348$, not adjusted for dropouts). It's no wonder noninferiority trials are not as common as placebo-controlled trials, as they often require much larger sample sizes. ${ }^{2}$

In the practical world of MS trials, current noninferiority trials are not using progression as their outcome variable because, as demonstrated, the sample sizes for such trials are enormous. Instead, these trials are using other outcome variables, such as enhancing lesion counts or possibly annualized relapse rates. These continuous outcomes or count data enable smaller sample sizes to be used, but the trial designer still needs to consider the careful justification of the tolerable difference.

Finally, a few cautions about noninferiority trials that are a bit counterintuitive should be mentioned. When investigators do a superiority trial, they continually explain to everyone that variance is the enemy. They try to standardize measurements and follow a protocol religiously in an effort to reduce extraneous sources of variability, because too much variability will make it unlikely that a treatment difference will be seen as statistically significant. Tests of the null hypothesis are based on the ratio of the difference between the treatments divided by the standard error of that difference. The standard error of the difference is driven by all the sources of variability in the data. If the denominator of this ratio has more variability because of sloppy and/or poor measurement and thus is larger than it should be, this makes the ratio of the mean difference to the standard error smaller than it should be or makes the $p$ value larger so that it doesn't meet the $<0.05$ level of significance.

In noninferiority trials, sloppiness biases the results away from the null hypothesis. ${ }^{1}$ Since the null hypothesis is that one treatment is worse than the other, sloppiness can make a trial successful when the treatments truly are not the same. For example, consider a poorly conducted study in which the pills are the size of apples and no one in either group takes the drug. Both groups have the same outcomes and there is no difference in the results. Because this is a truly equivalent outcome, the test of inferiority would be rejected and noninferiority would be concluded. It is true that in such a study the new treatment was not inferior to the old, but it has nothing to do with drug effects. While this is an extreme example, the methodology of a noninferiority trial is critical. The general rule to always use an intention-to-treat analysis does not in and of itself hold for these trials. ${ }^{3}$ Per-protocol analyses are also needed in order to assess the best estimates of the drug effects as well as the intention to treat. ${ }^{4}$

In summary, equivalence trials are useful to provide evidence so that one can trust the makeup and dosing of drugs from one manufacturer to another or from one batch to another. Noninferiority trials can provide comparisons between drugs that are thought to be equal in 
effectiveness, but they require added planning and decision making. ${ }^{5}$ Such trials need to justify the tolerable difference, set an expected outcome value (either the same or different between the test drug and the standard drug), and be very well-conducted so as not to bias the results away from the null hypothesis.

\section{REFERENCES}

1. Schumi J, Wittes JT. Through the looking glass: understanding non-inferiority. Trials 2011;12:106. doi: 10.1186/1745-6215-12-106.

2. Kaul S, Diamond GA, Weintraub WS. Trials and tribulations of non-inferiority: the ximelagatran experience. J Am Coll Cardiol 2005;46:1986-1995.

3. Wiens BL, Zhao W. The role of intention to treat in analysis of noninferiority studies. Clin Trials 2007;4:286-291.

4. Matilde Sanchez M, Chen X. Choosing the analysis population in non-inferiority studies: per protocol or intent-to-treat. Stat Med 2006;25:1169-1181.

5. Piaggio G, Elbourne DR, Altman DG, Pocock SJ, Evans SJ, Group C. Reporting of noninferiority and equivalence randomized trials: an extension of the CONSORT statement. JAMA 2006;295: $1152-1160$.

\section{STUDY FUNDING}

No targeted funding reported.

\section{DISCLOSURES}

G. Wang receives grant support from NIA and NARCOMS. G.R. Cutter has received grant or research support from participation on data and safety monitoring committees from Apotek, Biogen Idec, Cleveland Clinic, GlaxoSmithKline Pharmaceuticals, Gilead Pharmaceuticals, Modigenetech/Prolor, Merck/ Ono Pharmaceuticals, Merck, Neuren, PCT Bio, Revalesio, Sanofi-Aventis, Teva, Vivus, NHLBI (Bone Marrow Transplant Protocol Review Committee), National Institute of Neurological Disorders and Stroke, NMSS, and NICHD (OPRU oversight committee); has received consulting and/or speaker fees from Alexion, Allozyne, Bayer, Celgene, Coronado Biosciences, Consortium of MS Centers (grant), Diogenix, Klein-Buendel Incorporated, Immunotherapeutics, Medimmune, Novartis, Nuron Biotech, Receptos, Spiniflex Pharmaceuticals, Teva Pharmaceuticals, Merck, Opexa, Transparency Life Sciences, Nivalis Therapeutics, Inc., and Genzyme; is employed by the University of Alabama at Birmingham; is president of Pythagoras, Inc., a private consulting company located in Birmingham, AL; serves on the editorial board of Multiple Sclerosis and as a statistical consulting reviewer for JASN; receives no direct grants but works on studies funded to the Consortium of MS Centers subcontracted for analysis of NARCOMS Registry; receives/has received research support from the NIH (NIAID, NHLBI, NIA, National Institute of Neurological Disorders and Stroke, Consortium of MS Centers, and Myasthenia Gravis Foundation of America); and has reviewed statistical data for a medico-legal proceeding. Full disclosure form information provided by the authors is available with the full text of this article at Neurology.org/cp.

\section{What's New in Clinical Practice?}

Neurology ${ }^{\circledR}$ Clinical Practice now has podcasts available for download. The podcasts are introduced by Editor John R. Corboy, MD, FAAN, who highlights papers from the current issue. The interview that follows features authors discussing papers in more depth and bringing clinical implications to the fore. 